\title{
La Politique Salariale Et La Proletarisation Des Agents Et Fonctionnaires De L'etat. (Etude Appliquée Dans Les Services Publics De La Ville De Bukavu).
}

\author{
Mulumeoderhwa Bujiriri Le Bon ${ }^{1}$
}

\begin{abstract}
Résumé: Cet articule essai d'analyser la situation de misère traversée par les agents et fonctionnaires de l'Etat dans la ville de Bukavu. Cette situation s'explique par le fait que la politique salariale mise en place par l'Etat congolais est une politique non favorable à l'amélioration de leurs conditions de vie. Nombreux d'entre-eux travaillent sans salaire de l'Etat et d'autres qui en reçoivent, ils n'ont qu'un salaire insignifiant qui font d'eux des travailleurs pauvres. Ces agents et fonctions de l'Etat sont dans une misère exacerbée. Ainsi, ce phénomène est à la base de la corruption, la misère, le détournement des deniers publics, les retards et absences au travail,... des agents et fonctionnaires de l'Etat.
\end{abstract}

\section{INTRODUCTION}

Sur le marché du travail, le travail est considéré comme une marchandise avec son propre prix. Tout employé a droit à un salaire. Pour Muriel Maillefert, le travail humain n'est pas gratuit : "Le travail humain est une activité créatrice de richesse. Cette activité s'organise dans un cadre marchant.» ${ }^{2}$. Travailler c'est faire une allocation du temps. "Chaque individu dispose d'une dotation limitée de temps qu'il choisit de diviser entre le travail salarié et le loisir.» ${ }^{3}$. La rentabilité du travail exige de l'employeur une capacité d'organiser et d'exploiter rationnellement le temps du travail et les capacités du salarié. "La mise au travail salarial consiste en l'utilisation, par l'employeur, du temps et des capacité d'autrui, à des fins de production de biens et des services, sur la base d'une rémunération déterminée à l'avance ${ }^{4}$. Le salaire est une source clé du bien-être social pour tout travailleur. Il constitue l'élément primordial pour le travailleur, car c'est à partir de celui-ci que les besoins de l'employé sont satisfaits. C'est dans cette optique que l'Etat développe des mécanismes salariaux pour permettre aux agents et fonctionnaires d'avoir un salaire décent susceptible d'améliorer leurs conditions de vie. Dans la gestion stratégique des ressources humaines, le salaire constitue l'une des questions majeures dont tout employeur ne peut s'en passer.

La République Démocratique du Congo a traversé, pendant ces dernières décennies, plusieurs conflits de guerre et instabilités politiques qui ont affecté négativement les conditions de travail des agents et fonctionnaires de l'Etat. A cet effet, dans les services publics congolais, le salaire a perdu son caractère obligatoire et il est perçu comme une faveur pour les agents. C'est ainsi que ces agents reçoivent irrégulièrement un salaire inférieur à leur subsistance. Pourtant, le code du travail en RD Congo stipule que «le paiement de la rémunération doit être effectué à des intervalles réguliers n'excédant pas un mois. ${ }^{5}{ }^{\circ}$

D'autres agents et fonctionnaires ont travaillé depuis plusieurs années mais ils n'ont jamais reçu le salaire. Cette situation a soulevé tant de grognes dans le chef de ces agents et de temps à autre, on assistait à des grèves intempestives. Pour apaiser la situation, l'Etat a commencé à payer partiellement le salaire accumulant ainsi des arriérés qui ont plongé les agents et fonctionnaires dans la misère totale. Après les guerres de libération

\footnotetext{
${ }^{1}$ MULUMEODERHWA BUJIRIRI Le Bon, Licencié en Sociologie, Chercheur, Enseignant Chef des Travaux à l'ULGL/ Bukavu et Candidat finaliste pour la maîtrise en Sociologie à l'Université de Kisangani(UNIKIS)/RDC. Mail : lebonmulumeoderhwa@gmail.com Tél : +243 (993035496 853210014).

${ }^{2}$ M. MAILLEFERT, L'économie du travail. Concepts, débats et analyse (2éme édition), LevalloisPerret, Paris, 2004, p.11.

${ }^{3}$ C. PIERRE et ZYLBERBERG, Économie du travail. La formation des salaires et les déterminants du chômage, De Boeck, 1996, p.18.

${ }^{4}$ D. LINHART, Cité par G. DORE, Politique de formation professionnelle et de l'emploi en Haïti(le cas du secteur de tourisme 1980-2010), Thèse de doctorat en sciences de l'éducation, Université de Paris-Est, 2010, Inédit, p.118.

${ }^{5}$ Code du travail congolais, Kinshasa 2002, Art. 99. 
et des rebellions, l'Etat congolais a progressivement mis sur pieds de mécanismes d'amélioration de l'enveloppe et zones salariales de ses agents. Les barèmes salariaux ont été progressivement revus à la hausse, et c'est dans ce sens que l'Etat Congolais a signé des "accords de Mbudi », dont l'application effective pose encore problème jusqu'aujourd'hui. L'accord de Mbudi appelé encore contrat social de Mbudi ou contrat de l'innovation, est un accord signé le 12 février 2004 à Mbudi, une banlieue ouest de Kinshasa, entre le Gouvernement de transition de la RD Congo et les syndicats de l'Administration publique et interprofessionnels. Dans cet accord, le gouvernement congolais s'était engagé à payer à un huissier 208 \$US et au secrétaire général de l'Administration publique 2080 \$US. Depuis ce temps jusqu'aujourd'hui, cet accord n'a jamais été appliqué, les agents de l'Etat continuent à croupir dans la misère. Ces situations ne font qu'aggraver les conditions de vie de la masse laborieuse de la fonction publique.

Pour atteindre les objectifs de la présente recherche, nous nous posons principalement la question de savoir comment la politique salariale appliquée dans la fonction publique de la ville de Bukavu prolétise-t-elle les agents et fonctionnaires de l'Etat? Secondairement, quel serait l'impact de cette politique salariale sur les conditions de vie et de travail des agents et fonctionnaires de la Fonction publique de la ville de Bukavu ?

Provisoirement, nous retenons que la politique salariale dans la fonction publique de la ville de Bukavu prolétariserait les agents et fonctionnaires de l'Etat en ce sens qu'elle est inadaptée pour l'amélioration de conditions de vie de ces derniers qui reçoivent irrégulièrement un salaire dérisoire, accompagné de beaucoup d'arriérés impayés, où plusieurs d'entre-eux travaillent depuis plusieurs années sans salaire. L'impact de cette politique salariale serait de placer les agents et fonctionnaires de l'Etat dans une paupérisation exacerbée avec comme conséquence la corruption, les retards et absences au travail, etc.

\section{APPROCHE THEORICO-CONCEPTUELLE}

\section{II.1. Cadre conceptuelle}

\section{II.1.1. La politique salariale}

La politique salariale est d'importance capitale dans la gestion stratégique des ressources humaines recrutées au sein de toute entreprise. Elle va de pair avec la politique de l'emploi car on ne peut concevoir l'emploi sans salaire. En principe, le salaire devrait varier en fonction des coûts de vie (logement, alimentation, éducation,...). L'élaboration d'une échelle des salaires et l'application d'une politique de l'emploi suppose au préalable la maîtrise des effectifs. Elle implique la mise en place dans l'entreprise d'un échelonnement satisfaisant des postes les uns par rapport à d'autres, et d'un barème salarial correspondant. Jean-Marie Peretti ${ }^{6}$ note que la politique salariale repose sur la recherche d'un triple équilibre :

- l'équilibre financier de l'organisation ;

- la compétitivité externe compte tenu du marché du travail ;

- l'équité interne.

Pour ce qui est de la ville de Bukavu, il est difficile de confirmer qu'il existe la compétitivité salariale suite à l'existence d'une pénurie d'emplois. Au contraire, certains jeunes universitaires au chômage préfèrent quitter la ville de Bukavu pour la recherche de l'emploi dans d'autres pays fuyant le problème du chômage et espérant y trouver un emploi plus rémunérateur. La politique salariale a comme fonction de recruter des candidats répondants aux besoins réels de l'organisation, et constitue un moyen de reconnaissance vis-à-vis de l'employé par l'employeur.

\section{II.1.2. Travail, emploi et salaire \\ a). Le travail}

Le concept du travail implique la mise en action des aptitudes physiques et intellectuelles de l'homme dans le processus d'acquérir des biens susceptibles de satisfaire à un ou plusieurs besoins. Etant donné que le travail est pénible et demande un effort physique et/ou intellectuel, cet effort nécessite une rétribution ou alors une rémunération compensatrice. Dès lors, Guichard Doré ${ }^{7}$ note que le travail fait appel aux habiletés et aux capacités physiques et intellectuelles du salarié. Le travail, comme expression de la relation entre le corps et les objets pour la production d'un bien ou d'un service à valeur ajoutée, demande la mobilisation d'un capital de connaissances et d'expériences. Le travail entraîne des coûts pour l'entrepreneur et demande la mobilisation des énergies et des ressources immatérielles et parfois la mobilisation corporelle du salarié. Le travail fait appel à une double rationalité des acteurs impliquant dans le processus de production de biens et des services. Du côté

\footnotetext{
${ }^{6}$ J-M. PERETTI, PERETTI, Gestion des ressources humaines, Vuibert, Paris, 2005, p.148.

${ }^{7}$ G. DORE, Politique de formation professionnelle et de l'emploi en Haïti(le cas du secteur de tourisme 1980-2010), Thèse de doctorat, Université de Paris-Est, Sciences de l'éducation, Inédit, 2009-2010, pp.116-119. 
de l'employeur, il cherche à maximiser les profits de son entreprise en embauchant les salariés les plus efficaces au coût le plus bas possible. Du côté de l'employé, il cherche à avoir un emploi mieux rémunéré, si possible un salaire plus élevé, et de meilleures conditions de travail.

\section{b). L'emploi}

Selon Szczepanski, l'emploi implique une relation légalement définie entre employeur et employé dans laquelle deux éléments sont essentiels : l'exécution de tâches et sa rémunération ${ }^{8}$. L'emploi constitue une activité exercée par les agents et fonctionnaires de l'Etat dans le but d'acquérir un salaire nécessaire à la satisfaction de leurs besoins.

\section{c). Le salaire/ rémunération}

Le salaire est une rétribution, une somme d'argent donnée à un employé par son employeur après prestation des services selon les conventions interprofessionnelles. Il s'agit donc d'une récompense financière d'un service. Pour ce qui est de la rémunération, le code du travail ${ }^{9}$, dispose qu'elle est une somme représentative de l'ensemble des gains susceptibles d'être évalués en espèces et fixés par accord ou par les dispositions légales et réglementaires qui sont dus en vertu d'un contrat de travail, par un employeur à un travailleur. Elle comprend notamment : le salaire ou traitement, les commissions, l'indemnité de vie chère, les primes, la participation aux bénéfices, les sommes versées à titre de gratification ou de mois complémentaires, l'allocation de congé ou l'indemnité, compensatoire de congé, les sommes payées par l'employeur pendant l'incapacité de travail et pendant la période précédant et suivant l'accouchement.

\section{II.1.3. La prolétarisation}

Par prolétarisation, nous entendons ici le processus social tendant à l'appauvrissement de la classe ouvrière. Dans le cadre de cette étude, il s'agit de conditions de travail menant les agents et fonctionnaires de l'Etat vers une paupérisation aigue. Nous faisons usage de ce concept de prolétarisation, car les agents et fonctionnaires de l'Etat de la ville de Bukavu vivent dans des conditions extrêmement difficile suite au maigre salaire qu'ils reçoivent et d'autres travaillent sans même ce salaire.

\section{II.1.4. Les services publics}

Selon Charles Debbasch et Frédéric Colin ${ }^{10}$ services publics est une composante de l'administration publique dont la bonne marche permet la réalisation des objectifs définis par le pouvoir politique. Dans l'usage courant les notions d'administration publique et du service public sont de fois entremêler. L'administration a pour objectif essentiel de satisfaire l'intérêt général et la fonction publique est un des moyens dont elle dispose pour y parvenir. L'administration dispose de différents types de moyens pour assurer ses missions d'intérêt général: des moyens juridiques (ex : prérogatives de puissance publique), des moyens matériels (ex : le domaine public des personnes publiques comme les routes, les bâtiments publics...), des moyens humains (dont la fonction publique). La fonction publique est au service de l'administration. Elle comprend l'ensemble des fonctionnaires, c'est-à-dire l'ensemble des personnes nommées dans un emploi permanent et titularisées dans un grade de la hiérarchie des administrations de l'État. Le service public ${ }^{11}$ apparaît donc comme une fonction technique de l'administration, c'est-à-dire de l'ensemble des structures publiques ou privées chargées d'accomplir des activités d'intérêt général. Cependant, comme le mot administration, l'expression service public revêt plusieurs sens. Elle désigne d'une part, une activité ou une mission d'intérêt général (ex : service public de l'enseignement) et, d'autre part, l'ensemble des organismes en charge de ces activités d'intérêt général et qui peuvent être aussi bien publics que privés (ex : les sociétés d'autoroutes). On passe ainsi « du » service public «aux » services publics. Dans la Fonction publique congolaise, il existe 3 sortes de catégories de postes répartis selon les grades.

Tableau $\mathbf{n}^{\circ} \mathbf{1}$ : Répartition de poste selon le grade dans la fonction publique en R.D.C

\begin{tabular}{|l|l|}
\hline Catégorie de poste & Grade et nomination \\
\hline Poste de commandement & $110:$ Secrétaire général \\
& $120:$ Directeur \\
& $130:$ Chef de Division \\
& $140:$ Chef de Bureau \\
\hline Poste de collaboration & \\
\hline
\end{tabular}

\footnotetext{
${ }^{8}$ S ZCZEPASKI, cite par P. GROTINGS, « Les jeunes, le travail et l'emploi en Europe » In Jeunesse, mode de vie, travail et emploi, tendance de la recherche, UNESCO, n¹06, 1986, p.515.

${ }^{9}$ Art. 7 du Code du travail, loi n015/2002 du 15 octobre 2002, RDC.

${ }^{10} \mathrm{CH}$. DEBBASCH et F. COLIN, Administration publique (6 édition), Ed. Economica, Paris, 2005, P.1.

${ }^{11}$ Http : // www. Google. Com. Service public, consulté le 03 /06/2014 à 13h30'.

DOI: 10.9790/0837-2205044456 www.iosrjournals.org $\quad$ 46|Page
}


La Politique Salariale Et La Proletarisation Des Agents Et Fonctionnaires De L'etat. (Etude ..

\begin{tabular}{|l|l|}
\hline & $\begin{array}{l}210: \text { Attaché de Bureau de première classe } \\
220: \text { Attaché de Bureau de deuxième classe } \\
310: \text { Agent de Bureau de première classe }\end{array}$ \\
\hline Poste d'exécution & $\begin{array}{l}320: \text { Agent de Bureau de deuxième classe } \\
330: \text { Agent Auxiliaire de première classe } \\
340: \text { Agent Auxiliaire de deuxième classe } \\
350: \text { Huissier }\end{array}$ \\
\hline
\end{tabular}

Source : Journal Officiel de la République du Zaïre, Statut du personnel de carrière des services publics de l'Etat, n¹5, Août 1981, p.13.

Nous retenons dans ce tableau que dans la Fonction pulque en RDC, il existe 3 grandes catégories de poste (poste de commandement, poste de collaboration et poste d'exécution) réparties selon le grade. En principe, l'engagement des personnes au grade de commandement, c'est-à-dire aux grades 110, 120, 130 et 140 est souvent difficile pour faute d'expérience professionnelle des candidats. Ainsi, à chaque 3 ans de service, il est prévu une promotion, et c'est à partir de celle-ci que les agents et fonctionnaires de la Fonction publique peuvent aspirer à ce poste de commandement. Toutefois, la promotion ne se fait presque plus dans la Fonction publique car nombreux agents et fonctionnaires y sont pendant plus de 20 ans et leur grade reste statutaire ${ }^{12}$.

\section{II.2. TENTATIVE D'EXPLICATION THEORIQUE DU SALAIRE}

Pour expliquer la notion de salaire, plusieurs théories ont été développées. Pour mener à bon port cette étude, il nous est utile de décortiquer ces théories.

\section{II.2.1. Le salaire d'efficience}

La théorie du salaire d'efficience repose sur l'idée selon laquelle un employeur peut avoir intérêt à payer de hauts salaires afin d'encourager ses employés à être efficace au travail.

Quatre explications ont été avancées pour justifier une relation croissante entre salaire et productivité ${ }^{13}$ :

-Une politique de «bas salaires » favorise la rotation de la main d'œuvre et, par voie de conséquence, aura une influence négative sur la productivité du travail à moyen ou long terme.

-Un chef d'entreprise n'observant qu'imparfaitement les caractéristiques des individus qu'il embauche peut pratiquer une politique de « hauts salaires », afin d'attirer dans son entreprise les meilleurs éléments de la population active.

-Lorsque les actions des employés sont imparfaitement observées par l'employeur, il y a un problème d'aléa moral : les employés peuvent avoir intérêt à tirer au flanc. Payer de hauts salaires peut alors permettre d'inciter les employés à s'investir dans leur travail.

-Payer des salaires relativement élevés peut aussi constituer un moyen de signifier aux employés qu'ils sont traités équitablement, avec respect. Ceci peut favoriser des comportements coopératifs qui améliorent la productivité du travail.

Nombreux agents et fonctionnaires de l'Etat dans la ville de Bukavu sont démotivés suite à la modicité du salaire qu'ils reçoivent. Face à cette réalité, le rendement ne peut qu'être aussi médiocre. Pour y remédier, l'application de la théorie du salaire d'efficience s'avère indispensable pour améliorer non seulement les conditions de vie de ces agents mais aussi contribuer à la productivité dans différents services de la fonction publique.

Ainsi, la productivité de chaque salarié dépend de son effort, lequel croît avec le salaire versé. Pour les marginalistes, les travailleurs sont rémunérés selon leur productivité marginale. Aussi, l'entreprise doit savoir ce que les salariés ont produit afin de déterminer leur taux de rémunération ${ }^{14}$. Cette théorie nous impose le relativisme pour autant que dans la ville de Bukavu, la détermination ou alors l'augmentation du salaire des agents est aussi une question d'éthique et de morale (le souci de la justice distributive) et non seulement de la seule productivité. En effet, certaines entreprises peuvent connaître de progrès réel sans augmenter significativement le salaire de leurs employés. Le marché du travail à Bukavu est très déséquilibré de manière que les demandes sont plus supérieures par rapport aux offres. La conséquence directe de cette situation c'est la diminution du salaire pour les agents qui sont aliénés et réduits à une exploitation criante.

${ }^{12}$ Entretien réalisé avec un Responsable de la Fonction publique, le 20 janvier 2017 à $10 \mathrm{~h}$.

${ }^{13}$ G.TCHIBOZO, Cité par O.MARIKO, L'insertion professionnelle des diplômés de l'enseignement supérieur au Mali : Cas de la politique d'aide à l'entrepreneuriat, Thèse de doctorat en sciences économiques, Université de Grenoble, Inédit, 2011-2012, p.135.

${ }^{14}$ O.MARIKO, Op.cit, p.135. 


\section{II.2.2. Le modèle sociologique (Akerlof, 1984)}

Ce modèle analyse les contrats de salaire comme des "dons bilatéraux" entre l'entreprise et ses salariés dont l'efficacités productives dépendent en grande partie de leurs sentiments d'être " bien traités par l'employeur. Dans ces conditions, offrir un salaire supérieur au salaire de réservation constitue, de la part de l'employeur, une gratification susceptible d'inciter les travailleurs à fournir un niveau d'effort important. L'aspect sociologique » des considérations développées par Akerlof vient essentiellement du fait que les travailleurs adoptent, à l'égard de l'effort au travail, un comportement en partie dicte par ce qu'ils pensent être « la norme » du groupe auquel ils appartiennent.

Ainsi, si le salaire offert est supérieur à la norme, les travailleurs augmenteront leurs efficacités productives plus que le minimum requis. C'est pourquoi on parle d'échange de don (dons et contre-dons) : un salaire plus élevé contre davantage d'effort. Donc, l'efficacité productive du salarie est fonction du salaire perçu comme équitable, de l'effort demande par l'employeur.

Comme pour la théorie de salaire d'efficience, le modèle akrofien insiste sur l'augmentation du salaire des travailleurs dans l'entreprise pour la reconnaissance et l'augmentation de la productivité. Dans la ville de Bukavu, les agents et fonctionnaires de l'Etat reçoivent un salaire dérisoire qui ne peut leur permettre ni à répondre aux besoins fondamentaux de l'existence ni à améliorer la qualité des services rendus dans la fonction publique.

\section{II.3. L'approche marxiste du prolétariat \\ II.3.1. Le mode de production capitaliste}

Karl Marx développe le postulat selon lequel les conditions matérielles de reproduction d'une société sont jugées déterminantes ${ }^{15}$. Le MPC (Mode de Production Capitaliste) est une configuration particulière d'organisation sociale dans laquelle les rapports marchands tiennent une place déterminante.

Le MPC généralise les rapports marchands à tout l'espace social, y compris aux relations de travail. Le travail devient une marchandise. Le MPC se caractérise par trois éléments :

-la propriété privée des moyens de production ;

-la liberté formelle des agents économiques (salarié, capitaliste, rentier) ;

-le développement des rapports marchands.

Ce développement des rapports marchands masque la réalité du rapport de domination qui existe dans le système marchand et que l'économie dite vulgaire s'attache à ne pas reconnaître, en faisant croire que les catégories du MPC sont universelles.

La critique de l'économie politique se donne comme objectif de mettre à jour ce rapport de domination, qui existe principalement dans le domaine du travail : c'est la théorie de l'exploitation. En effet, dans la ville de Bukavu, l'Etat comme employeur, est propriétaire des moyens de production, il fixe d'autorité le salaire de ses employés et développe le rapport marchand d'«exploitation» au travers du salaire bas payé à ses agents et fonctionnaires. Ce salaire qui n'est même pas un salaire de subsistance enfoncerait nombreux agents et fonctionnaires dans la pauvreté et même dans l'hyper pauvreté. Dans ce contexte, il est difficile de considérer le travail comme un moyen de lutte contre la pauvreté. Pierre Bréchon note que «pour que le mode de production capitaliste se développe, il faut qu'en permanence des ouvriers salariés soient exploités par des capitalistes (...) et le travailleur étant toujours payé au niveau de subsistance, il ne peut jamais faire d'économie. Il ne peut pas accumuler du capital. Il est obligé de vendre sa force pour vivre. ${ }^{16}{ }_{\text {» }}$

\section{II.3.2. La valeur du travail et plus-value}

a). La valeur-travail

On sait que Marx s'appuie sur une théorie de la valeur travail. Selon lui, le travail est fondement et mesure de la valeur. Ainsi, la valeur d'un bien est la quantité de travail abstrait socialement nécessaire à la production de ce bien. Cette même théorie s'applique au travail comme marchandise. Le salarié met à la disposition de l'employeur sa force de travail qui a une valeur : la quantité de travail nécessaire à sa production, c'est-à-dire la valeur des biens nécessaires à l'entretien du salarié et de sa famille ${ }^{17}$. Nous notons ici que le travail est payé à sa valeur : l'échange marchand est un échange d'équivalents. Mais la force de travail est une marchandise particulière : sa consommation crée de la valeur. En effet, supposons que pour payer les biens nécessaires à son entretien, un ouvrier doit travailler huit heures par jour. Le temps de travail légal est de dix

${ }^{15}$ G. Abraham-François, Cité par O. MARIKO, Op.cit, p.104.

${ }^{16}$ P. BRECHON, Les grands courants de la sociologie, PUG (Presse Universitaire de Grenoble), Paris, 2000, p.39.

${ }^{17}$ M. MAILLEFERT, Cité par OUSMANE MARIKO, Op.cit, p.114. 
heures. Il y a donc deux heures de travail non rémunérées que le capitaliste s'approprie. Ce temps est le « surtravail ».

Ainsi peut-on expliquer la métamorphose de la valeur : le capitaliste achète et vend des marchandises à leur valeur, mais en retire quand même un profit, qui ne se réalise cependant qu'une fois le bien vendu. Sur le plan social, la valeur du travail c'est de permettre l'intégration sociale de l'employé.

Pour ce qui est du travail des agents et fonctionnaires de l'Etat dans la ville de Bukavu, il est difficile de vite conclure que leur travail est une valeur au sens marxiste pour autant que ces derniers reçoivent un salaire insignifiant ne leur permettant pas une véritable intégration sociale.

\section{b). La plus-value}

Karl Marx distingue le travail, dont la quantité est mesurée en heures de travail, et d'autre part la force de travail, dont la valeur est donnée par la quantité de travail qui est incluse dans les biens et services que le travailleur consomme. Considérez le travailleur lui-même comme une sorte de machine dans laquelle on enfourne des biens et services, et à la sortie de la machine, cela produit de la force de travail. La force de travail est le résultat d'un processus de production. D'un côté vous mettez du pain, de l'eau, des habits, un logement, bref de quoi satisfaire les besoins élémentaires d'un être humain, et de l'autre vous obtenez une marchandise qui est la force de travail, et cette force de travail, comme toute marchandise, est soumise à la loi de la valeurtravail, c'est-à-dire que sa valeur est égale à la quantité de travail « socialement nécessaire ». Autrement dit la quantité en moyenne nécessaire pour élever, nourrir, loger le travailleur et satisfaire à ses besoins sexuels et sa reproduction $^{18}$.

Le patron tire du travailleur une quantité de travail toujours supérieure à la valeur de la force de travail. Cette différence, c'est la plus-value. C'est ce que pense Ahmed Silem et ses collaborateurs lorsqu'ils préconisent que la plus-value est l'augmentation de la valeur, produite par le travail de l'ouvrier salarié et que s'approprie le capitaliste gratuitement ${ }^{19}$. Sans chercher à entrer en contradiction avec les auteurs ci-haut cités, nous considérons la plus-value comme une quantité du travail non rémunérée à un ouvrier salarié par l'employeur.

Exemple : Si la valeur de la force de travail est de 6 heures par jour et que le capitaliste (employeur) emploie le salarié 8 heures, il le payera 6 heures, les marchandises seront vendues à l'équivalence de 8 heures; la différence de 2 heures constituent la plus-value. Elle résulte à cet effet d'une exploitation du travail du salarié. Dans le contexte de la théorie de la plus-value, il est difficile pour un travailleur d'accéder à un salaire d'efficience susceptible de promouvoir son investissement sinon, il ne se contentera que de son salaire de subsistance pour le renforcement de sa force productive.

Pourquoi y aurait-il toujours une différence entre la valeur de la force de travail et la quantité de travail effectuée par les travailleurs ? C'est la difficulté de la théorie. Il y a deux manières d'y répondre : par la loi d'airain des salaires, et par la théorie de la coalition des patrons ${ }^{20}$.

\section{II.3.3. Les lois de paupérisation}

La concurrence entre les salariés (et entre capitalistes) ainsi que la pression démographique maintiennent les salaires de longue période au minimum vital : c'est la « loi d'airain des salaires », adoptée par tous les classiques. La loi d'airain des salaires ${ }^{21}$ est une formule de Ferdinand Lassalle, auteur socialiste allemand (1825-1864) selon laquelle le salaire ne dépasse pas le minimum vital nécessaire à la subsistance. Sous le nom exact de «loi d'airain économique », reprenant la loi de Ricardo ainsi que celle de Marx, Lassalle constatait que la valeur de la force de travail, telle d'une marchandise, dépend de son coût de production, c'est-à-dire l'ensemble des sommes nécessaires à l'entretien de la vie de l'ouvrier et à la perpétuation de son espèce. Le salaire ne peut durablement s'élever au-dessus de ce niveau car l'accroissement du nombre des ouvriers, par suite de l'amélioration de leur existence et de la concurrence qui en résulterait, le ramènerait à son taux primitif. Il ne peut non plus longtemps être inférieur car l'émigration, le célibat, la réduction du nombre des naissances, diminueraient les nombres des ouvriers et, par concurrence, ramèneraient les salaires à son taux antérieur. Ce modèle consiste à dire que les ouvriers se faisant concurrence pour offrir leur force de travail, les salaires ne feront que diminuer. Marx avait d'ailleurs refusé cette loi, la considérant à juste titre comme insultante à l'égard de la classe ouvrière, pour la remplacer par la théorie de la coalition des patrons afin de justifier la théorie du salaire de subsistance. Or, ce dont la « loi d'airain » ne tient pas compte, c'est que les patrons sont exactement dans la même situation face aux ouvriers : les patrons se concurrencent

${ }^{18}$ O. MARIKO, Op.cit, p.114.

${ }^{19}$ A. SILEM, Lexique d'économie (11 ${ }^{\mathrm{e}}$ édition), Dalloz, Paris, 2010, p.639.

${ }^{20}$ Idem.

${ }^{21}$ J-P. HUET, « loi d'airain » In Encyclopédia universalis, (En ligne), Consulté le 24 février 2017. http://www.Universalis.fr/encyclopedie/loi d'airain. 
pour débaucher et employer les ouvriers, ce qui permet à ces derniers de discuter du niveau des salaires et de faire jouer la concurrence entre patrons.

On voit donc que plus il y a de patrons, plus important est le moyen de pression des ouvriers sur ceuxci : Cette loi part du principe selon lequel : «Si tu ne veux pas m'augmenter le salaire, alors je pars à hier». Selon nous, ce modèle théorique, quoi que valide ne prend pas du tout en compte le contexte particulier du marché de travail où évolue les agents et fonctionnaires de l'Etat à Bukavu. En effet, dans un pays comme la RDC, il est très difficile même de trouver un emploi où on gagnerait juste un salaire de subsistance. Le marché du travail à Bukavu fonctionne de telle sorte que même l'accès à un salaire de subsistance est une aubaine pour nombreuses personnes réduites au chômage. Suite à la rareté des patrons et à l'incapacité pour ces derniers de créer un emploi décent aux chômeurs, il sera difficile pour un chômeur recruté d'abandonner son poste. Voilà ce qui fait que nombreux agents et fonctionnaires de l'Etat restent campé à leur poste malgré le maigre salaire par manque où aller. Bref, cette loi ne peut s'appliquer dans un milieu comme la ville de Bukavu où il est difficile de trouver de l'emploi. Au contraire, on y observe plus ce que nous pouvons appeler l'immobilisme professionnel, c'est-à-dire la situation où les agents et fonctionnaires acceptent tacitement, l'exploitation de leur patron. Ils admettent de rester dans leur profession quelle que soit le retard dans le payement du salaire dérisoire. Ces agents sont aliénés par leur propre activité professionnelle qui les place dans la pauvreté. Cependant, on peut se poser la question de savoir quel est le rôle du syndicat dans ce contexte.

Ainsi, la société tend à se polariser en deux groupes sociaux, les travailleurs et les capitalistes (détenteurs des moyens de production), car les indépendants (artisans et petits paysans) font faillite et viennent grossir les rangs des salariés ${ }^{22}$.

Ainsi, les entreprises cherchent-elles sans cesse à augmenter la plus-value :

-soit de manière absolue, en allongeant la durée du travail ;

-soit de manière relative, en cherchant à augmenter la productivité du travail (par exemple en investissant dans des machines plus sophistiquées). A ce moment, la valeur de la force de travail diminue car il faut moins de travail pour produire un bien.

\section{II.3.4. La coalition des patrons}

Les capitalistes louent aux prolétaires leur force de travail, et se constituent en cartel pour éliminer entre eux la concurrence sur le marché du travail et maintenir ainsi le salaire au plus bas niveau possible. Et ce plus bas niveau possible ne peut être que le salaire de subsistance. Dans ce point de vue, l'épargne et l'investissement du salarié n'est du tout possible, et donc son travail est précaire et ne peut lui assurer une véritable intégration sociale. C'est pourquoi, à l'égard de la théorie de la coalition des patrons, Karl Marx propose aux travailleurs de s'unir pour le renversement de la pyramide. C'est peut être l'une des stratégies envisageables mais le processus ne pourra aboutir du coup, car dans la ville de Bukavu, les travailleurs se regroupent en syndicats mais souvent leurs demandes ne sont pas prises en compte et leurs actions sur le terrain restent encore très limités. Nous pouvons ajouter en disant que la coalition des patrons s'explique par le dysfonctionnement du marché du travail lié aussi à la volonté des patrons, c'est-à-dire l'Etat congolais comme pourvoyeurs d'emplois et de salaire décent. Suite à ce dysfonctionnement du marché du travail, et dans la perspective d'exploitation, l'Etat fixe à son gré le salaire des agents et fonctionnaires, et cela sans application stricte de la législation du travail et d'autres textes légaux.

\section{II.3.5. Le salaire de subsistance et la prolétarisation}

Selon Karl Marx ${ }^{23}$, le salaire tend vers un niveau de subsistance assurant la reproduction de la force de travail. Ce niveau n'est pas défini une fois pour toutes. Il est « socialement déterminé » par les conditions de vie de chaque époque. Et la logique capitaliste de maximisation du profit conduit à minimiser le salaire permettant cette reproduction.

Toutefois, Marx n'accepte pas l'idée que ce salaire soit « naturel ». Pour lui, il ne s'agit que d'une règle sociale, historique, caractéristique du mode de production capitaliste. D'autre part, selon Marx, la réduction du salaire à son niveau de subsistance n'empêche nullement le chômage car celui-ci est, au contraire, le moyen mis en œuvre par les capitalistes pour faire baisser les salaires : plus il y a de chômeurs, moins les travailleurs seront exigeants (c'est la fameuse thèse de l' « armée industrielle de réserve »). Il est difficile de parler même d'un salaire de subsistance pour les agents et fonctionnaires de l'Etat étant donné que ces derniers reçoivent un salaire qui ne peut leur permettre de résister face aux conditions de vie difficile dans la ville de Bukavu.

\footnotetext{
${ }^{22}$ M. MAILIFFERT, Cité par O. MARIKO, Op.cit, p.116.

${ }^{23}$ A. PATRICK et M. ALAIN-PIERRE, Cité par M.SUM, Op.cit, p.67. 


\section{II.3.6. L'aliénation du travail dans la littérature sociologique}

Selon Jean Etienne et ses collaborateurs ${ }^{24}$, le thème de l'aliénation dans le travail qui a été le plus largement étudié par Marx met en évidence quatre dimensions du phénomène :

- L'ouvrier est d'abord aliéné par rapport au produit de son travail dont il est dépossédé après l'avoir fabriqué. Plus il produit, plus il s'appauvrit et moins il peut satisfaire à ses besoins humains.

- L'ouvrier est également aliéné par rapport à l'activité de travail elle-même. Parce qu'il ne contrôle pas le processus de production qui est dirigé par le capitaliste. Le travail perd donc pour lui toute signification. Ce travail forcé « ruine son esprit et meurtrit son corps».

- $\quad$ Il est de même aliéné par rapport au genre humain. En effet, à la différence de l'animal, l'homme ne produit pas seulement pour satisfaire ses besoins mais aussi pour se réaliser. Le travail pour lui est naturellement une activité libre, consciente et créatrice. En transformant la nature, il se transforme lui-même et accomplit ses potentialités d'homme.

- Enfin, l'ouvrier est aliéné par rapport aux autres hommes : par rapport au capitaliste qui exploite et ne voit en lui qu'un moyen pour augmenter sa plus-value, mais aussi par rapport à ses compagnons de travail, avec lesquels il se trouve en concurrence sur le marché du travail.

Dans le même ordre d'idées, on doit à l'américain Melvin Seeman d'avoir tenté de décomposer la notion d'aliénation en cinq dimensions ${ }^{25}$ :

- L'impuissance : qui est le sentiment de ne pas pouvoir contrôler les résultats de son activité, d'être dans l'incapacité d'agir sur son propre destin, d'avoir prise sur le cours de choses.

- L'imprécision de non-sens : liée à l'absence de signification de ce que l'on fait, à l'incapacité à comprendre le sens de la totalité des processus d'une division du travail toujours plus complexe.

- L'anomie : l'absence des normes identifiables, mais aussi, au sens de Merton, l'inadaptation de ces normes aux buts valorisés par la société, dont l'emploi fait partie.

- L'isolement social : se caractérise par le fait que l'individu devient étranger au monde, et plus spécialement à la société à laquelle il appartient. A cet effet, le chômage constitue donc un facteur déterminant de la désintégration sociale des jeunes universitaires.

- Le sentiment d'être étranger à soi-même : ne pas reconnaître dans ce que l'on fait ou dans ce que l'on est.

Ainsi, nous pouvons dire que l'aliénation du travail présente des caractéristiques liées au manque, par l'individu, le contrôle de son travail, l'ignorance de la fonction du travail comme facteur de transformation personnelle et sociale, et l'absence de l'intégration sociale par le travail. Elle est une manifestation d'une situation anomique de la division du travail. De ce fait, elle est le produit du manque réel de la solidarité sociale pour l'intégration sociale des membres de la société. Par exemple, un salarié qui produit dans une entreprise et qui ne reçoit qu'un salaire de subsistance ou non est aliéné par le même travail.

\section{PRESENTATION, ANALYSE ET INTERPRETATION DES RESULTATS} III.1. Milieu, matériel et méthodologie

\section{III.1.1. Milieu}

La ville de Bukavu est le chef-lieu de la province du Sud-Kivu appelée jadis Constermansville, elle a été créée par les Colons Belges sur l'ordonnance $n^{\circ} 12 / 357$ du 6 Septembre 1956. Elle est géographiquement située à l'extrémité Sud du lac Kivu à $28^{\circ} 30^{\prime}$ et $28^{\circ} 51^{\prime}$ de longitudes Est, et entre $2^{\circ} 26^{\prime}$ et $2^{\circ} 33^{\prime}$ de latitude Sud, à une altitude moyenne de 1650 mètres. Elle demeure jusqu'ici la plus haute de la RDC devant celle de Goma qui est à 1493 mètres d'altitude.

Elle est limitée :

- Au Nord par le lac Kivu

-Au Sud et à l'ouest par le territoire de Kabare

- A l'Est par la rivière Ruzizi qui sépare la RDC du Rwanda.

Elle est subdivisée en trois communes (Kadutu, Ibanda et Bagira) dont chacune est subdivisée en quartiers, avenues, sentiers, etc. Chaque commune regorge des services publics de l'Etat dans lesquels nous avons effectué nos enquêtes.

\section{III.1.2. Matériels}

La réalisation de cette étude a été rendue possible grâce aux matériels dont voici : les stylos, les papiers, le carnet de bord, le questionnaire d'enquête, la calculatrice, l'ordinateur, l'imprimante, les manuels scientifiques, etc.

\footnotetext{
${ }^{24}$ J. ETIENNE et alii, Dictionnaire de sociologie, Hatier, Paris, 2007, pp.24-27.

25 J. ETTIENNE, Op.cit., p. 27. 


\section{III.1.3. Méthodologie}

Par méthodologie, nous entendons ici la démarche pour saisir objet, la méthode et les techniques utilisées pour la collecte et le traitement des données. La méthode en soit est un ensemble des opérations intellectuelles permettant d'analyser, de comprendre et d'expliquer la réalité étudiée. Le présent travail a été matérialisé grâce à la méthode dialectique de Karl Marx. Elle est, selon Mascotch Nday Wa Mande, une double démarche à la fois théorique et pratique. La démarche théorique est un corps de principes qui oriente l'activité pensante et la manière de réunir les informations, tandis que la démarche appliquée donne un cadre d'application des principes et présupposés théoriques à l'objet de la recherche en vue 'atteindre les objectifs qu'on s'est assigné au départ ${ }^{26}$.

Selon son protocole descriptif, l'application de cette méthode obéit a quatre lois fondamentales :

- La loi de la connexion universelle des phénomènes; avec ce principe, nous allons présenter la politique salariale dans l'interconnexion avec d'autres phénomènes qui l'environnent notamment les conjonctures politique, économiques et socio-demographiques en RDC.

- La loi de changement de la quantité en qualité selon laquelle les phénomènes sociaux changent et se conservent les uns dans les autres. Cette loi nous permettra d'examiner si le salaire perçu par les agents et fonctionnaires de l'Etat dans les services publics, permet à ces derniers d'améliorer quantitativement et qualitativement leur condition de vie.

- La loi de la contradiction selon laquelle, les faits sociaux sont des unités de contraire. Dans cette loi, nous montrons comment les agents et fonctionnaires de l'Etat sont des «travailleurs pauvres» au regard de leur salaire qu'ils perçoivent.

- La loi de lutte de contraire selon laquelle les faits en opposition perpétuelle. Nous analysons les contrastes observés dans les accords salariaux et la réalité pratique sur le terrain en faveur des agents et fonctionnaires de l'Etat.

Cette méthode a été appuyée par la technique documentaire, d'entretien et de questionnaire. Nous avons aussi fait usage de la technique d'échantillonnage par quotas ou à choix raisonné. Etant donné qu'il nous a semblé difficile d'enquêter tous nos enquêtés, compte tenu des contraintes temporelles, matérielles et financières, notre échantillon a été de 80 personnes de 40 services publics ciblés à Bukavu.

En effet, nous avons d'abord construit un modèle réduit de l'univers de notre enquête composé de $\mathbf{3 . 6 1 3}$ agents et fonctionnaires de l'Etat. Ensuite, dans le cadre de ce modèle (plan d'enquête), nous avons déterminé les quotas, c'est-à-dire les catégories de personnes à enquêter dans chaque service public ciblé. Ainsi, sur l'ensemble de 3.613 agents et fonctionnaires identifiés, nous en avons enquêté 80, soit 2 enquêtés par service.

III.2. De la prolétarisation des agents et fonctionnaires de l'Etat par la politique salariale

Dans les services publics de la ville de Bukavu, nombreux agents et fonctionnaires de l'Etat travaillent sans recevoir un salaire et d'autres ne reçoivent qu'un salaire minimum à leur subsistance.

Tableau $\mathbf{n}^{\circ} 2$ : Statistique des agents et fonctionnaires « Sous-statuts », payés, impayés et Nouvelles Unités (N.U) de l'Etat contrôlés biométriquement en mai-juin 2013 à Bukavu

\begin{tabular}{|c|c|c|c|c|c|}
\hline $\mathrm{N}^{\circ}$ & Dénomination des services & $\begin{array}{l}\text { Cadre } \\
\text { organique }\end{array}$ & $\begin{array}{ll}\begin{array}{l}\text { Sous } \\
\text { payés }\end{array} & \text { statuts } \\
\end{array}$ & $\begin{array}{l}\text { Sous statuts } \\
\text { impayés }\end{array}$ & $\begin{array}{l}\text { Nouvelles } \\
\text { unites }\end{array}$ \\
\hline $\mathrm{O} 1$ & Action humanitaire & 70 & 3 & 0 & 61 \\
\hline 02 & Affaires sociales & 1129 & 154 & 100 & 541 \\
\hline 03 & Anciens combatants & 44 & 11 & 0 & 25 \\
\hline 04 & Assemblée provincial & 52 & 10 & 13 & 61 \\
\hline 05 & Brigade de la police judiciaire & 286 & 33 & 39 & 21 \\
\hline 06 & Budget & 139 & 23 & 87 & 86 \\
\hline 07 & Cadastre/ Bukavu & 0 & 10 & 29 & 56 \\
\hline 08 & $\begin{array}{ll}\text { Circonscription foncière/ } \\
\text { Bukavu }\end{array}$ & 0 & 32 & 19 & 75 \\
\hline 09 & Commerce extérieur & 269 & 71 & 63 & 9 \\
\hline 10 & Communication et medias & 52 & 19 & 22 & 100 \\
\hline 11 & Cour d'appel et des tribunaux & 394 & 19 & 17 & 64 \\
\hline 12 & Culture et art & 214 & 71 & 50 & 124 \\
\hline 13 & $\begin{array}{l}\begin{array}{l}\text { Décentralisation et affaire } \\
\text { coutumière }\end{array} \\
\end{array}$ & 171 & 13 & 22 & 17 \\
\hline
\end{tabular}

\footnotetext{
${ }^{26}$ M. NDAY WA MANDE, Memento des méthodes de recherché en sciences sociales et humaines, Zoé créativité, Likasi, 2004, p.29.
} 
La Politique Salariale Et La Proletarisation Des Agents Et Fonctionnaires De L'etat. (Etude ..

\begin{tabular}{|c|c|c|c|c|c|}
\hline & Droits humains & 0 & 0 & 7 & 94 \\
\hline 15 & Economie nationale & 186 & 48 & 29 & 17 \\
\hline 16 & Emploi et travail & 0 & 7 & 1 & 17 \\
\hline 17 & Energie & 82 & 5 & 8 & 459 \\
\hline 18 & $\begin{array}{l}\text { Environnement, } \\
\text { conservation nature }\end{array}$ & 960 & 87 & 71 & \\
\hline 19 & Finances & 133 & 50 & 65 & 21 \\
\hline 20 & Fonction publique/ Actifs & 63 & 41 & 16 & 16 \\
\hline 21 & Fonction publique/Passifs & 141 & 15 & 28 & 15 \\
\hline 22 & Genre, famille et enfant & 517 & 15 & 17 & 69 \\
\hline 23 & Habitat & 150 & 39 & 46 & 63 \\
\hline 24 & Hydrocarbures & 22 & 2 & 3 & 10 \\
\hline 23 & Industrie & 40 & 21 & 69 & 78 \\
\hline 24 & Inspection du travail & 0 & 9 & 1 & 17 \\
\hline 25 & Intérieur, sécurité & 686 & 625 & 428 & 138 \\
\hline 26 & Jeunesse & 541 & 56 & 17 & 79 \\
\hline 27 & Justice, garde des sceaux & 290 & 41 & 79 & 44 \\
\hline 28 & Mines & 213 & 43 & 196 & 217 \\
\hline 29 & $\begin{array}{l}\text { Parquet générale, grande } \\
\text { instance }\end{array}$ & 96 & 67 & 26 & 46 \\
\hline 30 & Parquet civil & 157 & 40 & 50 & 87 \\
\hline 31 & P.M.E & 615 & 37 & 32 & 180 \\
\hline 32 & Plan & 39 & 14 & 10 & 102 \\
\hline 33 & P.T.T & 79 & 3 & 3 & 36 \\
\hline 34 & Prévoyance sociale & 157 & 2 & 16 & 39 \\
\hline 35 & Reconstruction & 43 & 9 & 15 & 17 \\
\hline 36 & Sports et loisirs & 172 & 52 & 16 & 70 \\
\hline 37 & Tourisme & 144 & 70 & 22 & 54 \\
\hline 38 & $\begin{array}{l}\text { Transports et voies de } \\
\text { communication }\end{array}$ & 324 & 244 & 150 & 224 \\
\hline 39 & $\begin{array}{l}\text { Travaux publics et } \\
\text { infrastructures }\end{array}$ & 672 & 313 & 30 & 146 \\
\hline 40 & Urbanisme & 117 & 33 & 70 & 18 \\
\hline \multicolumn{2}{|c|}{ TOTAL GENERAL } & 9.459 & 2.457 & 1.982 & 3.613 \\
\hline
\end{tabular}

Source : Archives de la Division de la Fonction publique chargée des actifs/Sud-Kivu

Ce tableau indique que l'Administration publique de la ville de Bukavu prévoit environ 9.459 postes pour les personnes--- à recruter dans différents services ci-haut cités hormis certains services à l'exemple du travail et emploi, Doits humains, Circonscription foncière, Cadastre et Inspection du travail dont leurs cadres organiques ne nous étaient pas précisés. Ainsi, sur l'ensemble de 9.459 personnes à recruter par l'Administration publique, 1.407 postes restent à pourvoir. Bien plus, sur le total de 8.052 travailleurs dans les services de l'Administration publique, 2.457 soit 30,5\% sont reconnus comme agents sous statuts et qui reçoivent le salaire de l'Etat ; 1.982 soit $24,6 \%$ sont sous statut (ont le numéro matricule) mais ils ne sont pas payés par leur employeur qui est l'Etat congolais. De ces 8.052 effectifs, 3.613 soit 44,8\% ont été recensés comme Nouvelles Unités au sein de l'Administration publique de la ville de Bukavu; ils travaillent comme agents de l'Etat mais ne reçoivent pas le salaire de l'Etat.

Pour bien élucider l'ampleur du phénomène de la prolétarisation des agents et fonctionnaires de l'Etat dans la ville de Bukavu, il est impérieux de présenter l'évolution de la paie des agents de la Fonction publique de la ville de Bukavu. 
La Politique Salariale Et La Proletarisation Des Agents Et Fonctionnaires De L'etat. (Etude ..

Tableau n $\mathbf{n}^{\circ}$ : Etat d'évolution du salaire des agents et fonctionnaires de la Fonction publique à Bukavu

\begin{tabular}{|c|c|c|c|c|c|c|}
\hline $\mathrm{N}^{\circ}$ & Grades statutaires & Année 2009 & Année 2010 & Année 2011 & Année 2012 & Année 2013 \\
\hline 01 & Directeur & $26.405,00 \mathrm{FC}$ & $40.905,00 \mathrm{FC}$ & $59.923,00 \mathrm{FC}$ & $64.523,00 \mathrm{FC}$ & $74.923,00 \mathrm{FC}$ \\
\hline 02 & Chef de Division & $26.165,00 \mathrm{FC}$ & $40.665,00 \mathrm{FC}$ & $57.430,00 \mathrm{FC}$ & $62.430,00 \mathrm{FC}$ & $72.430,00 \mathrm{FC}$ \\
\hline 03 & Chef de Bureau & $25.448,00 \mathrm{FC}$ & $39.948,00 \mathrm{FC}$ & $55.403,00 \mathrm{FC}$ & $60.403,00 \mathrm{FC}$ & $70.403,00 \mathrm{FC}$ \\
\hline 04 & $\begin{array}{l}\text { Attaché de Bureau } \\
1^{\text {ère }} \text { classe }\end{array}$ & $23.532,00 \mathrm{FC}$ & $38.032,00 \mathrm{FC}$ & $51.564,00 \mathrm{FC}$ & $56.564,00 \mathrm{FC}$ & $66.564,00 \mathrm{FC}$ \\
\hline 05 & $\begin{array}{l}\text { Attaché de Bureau } \\
\text { de } 2^{\text {eme }} \text { classe }\end{array}$ & $22.722,00 \mathrm{FC}$ & $37.222,00 \mathrm{FC}$ & $49.956,00 \mathrm{FC}$ & $54.956,00 \mathrm{FC}$ & $64.956,00 \mathrm{FC}$ \\
\hline 06 & $\begin{array}{l}\text { Agent de Bureau de } \\
1^{\text {ere }} \text { classe }\end{array}$ & $22.096,00 \mathrm{FC}$ & $36.596,00 \mathrm{FC}$ & $48.692,00 \mathrm{FC}$ & $53.692,00 \mathrm{FC}$ & $63.692,00 \mathrm{FC}$ \\
\hline 07 & $\begin{array}{l}\text { Agent de Bureau de } \\
2^{\text {eme }} \text { classe }\end{array}$ & $21.698,00 \mathrm{FC}$ & $36.198,00 \mathrm{FC}$ & $47.892,00 \mathrm{FC}$ & $52.892,00 \mathrm{FC}$ & $62.892,00 \mathrm{FC}$ \\
\hline 08 & $\begin{array}{l}\text { Agent auxiliaire } 1^{\text {ère }} \\
\text { classe }\end{array}$ & $21.378,00 \mathrm{FC}$ & $35.878,00 \mathrm{FC}$ & $47.256,00 \mathrm{FC}$ & $52.256,00 \mathrm{FC}$ & $62.256,00 \mathrm{FC}$ \\
\hline 09 & $\begin{array}{l}\text { Agent auxiliaire de } \\
2^{\text {eme }} \text { classe }\end{array}$ & $21.138,00 \mathrm{FC}$ & $35.638,00 \mathrm{FC}$ & $40.638,00 \mathrm{FC}$ & $45.638,00 \mathrm{FC}$ & $55.638 \mathrm{FC}$ \\
\hline 10 & Huissier & $20.500,00 \mathrm{FC}$ & $35.000,00 \mathrm{FC}$ & $20.500,00 \mathrm{FC}$ & $20.500,00 \mathrm{FC}$ & $30.500,00 \mathrm{FC}$ \\
\hline
\end{tabular}

Source : Archives de la Division provinciale de la Fonction publique chargée des actifs/Sud-Kivu.

Ce tableau éclaircit comment le salaire des agents et fonctionnaires de l'Etat a évolué en fonction des années et des grades statutaires de chaque agent. Si on s'en tient à cette évolution, aujourd'hui un Chef de Division a un salaire mensuel d'environs 78.000FC, soit 60 \$USD ; le chef de Bureau 7.600FC, soit 58,4 \$USD; l'Attaché de Bureau de première classe 72.000FC, soit 55,3 \$USD; l'Attaché de Bureau de deuxième classe 70.000FC,soit 53,8 \$USD; l'Agent de Bureau de première classe 69.000FC, soit 53 \$US ; l'Agent de Bureau de deuxième classe 68.000FC, soit 52,3 \$USD; l'Agent auxiliaire de première classe 67.000FC, 51,5 \$USD; l'Agent auxiliaire de deuxième classe 66.000FC, soit 50,7 \$USD ; et au Huissier 63.000FC, soit 48,4 \$USD. Donc dans les services publics de la ville de Bukavu, aucun agent ne reçoit un salaire de 100 \$USD. A notre avis, cela paraît pour nous un salaire de misère et de la prolétarisation des agents et fonctionnaires de l'Etat, car avec la conjoncture économique actuelle, ce salaire ne peut permettre à ces derniers de satisfaire à ses besoins de base.

Tableau $\mathbf{n}^{\circ}$ 4: Répartition des enquêtés selon la réception régulière de leurs salaires

\begin{tabular}{|l|c|l|}
\hline $\begin{array}{l}\text { Recevez-vous régulièrement } \\
\text { votre salaire? }\end{array}$ & Effectifs & Pourcentage \\
\hline Oui & 13 & 16,2 \\
\hline Non & 62 & 77,5 \\
\hline Neutre & 5 & 6,2 \\
\hline Total & 80 & 100 \\
\hline
\end{tabular}

Source : nos enquêtes sur le terrain

Il ressort de ce tableau que sur l'effectif de 80 personnes enquêtées, 62 d'entre-elles, soit 77,5\% disent recevoir irrégulièrement leur salaire contre 13 , soit $16,2 \%$ qui pensent en recevoir à temps. 5 enquêtés, soit $6,2 \%$ sont restés neutre sur la question et ne se sont pas prononcés.

Tableau $\mathbf{n}^{\circ}$ 5: Classification des enquêtés selon que le salaire leur permet de répondre aux besoins vitaux

\begin{tabular}{|l|c|l|}
\hline $\begin{array}{l}\text { Votre salaire vous permet-il de } \\
\text { répondre à vos besoins vitaux? }\end{array}$ & Effectifs & Pourcentage \\
\hline Oui & 2 & 2,5 \\
\hline Non & 78 & 97,5 \\
\hline Total & 80 & 100 \\
\hline
\end{tabular}

Source : nos recherches sur le terrain

De ce tableau, il se dégage que sur l'ensemble de 80 personnes enquêtées, 78 soit 97,5\% nous ont confirmé que le salaire qu'ils reçoivent est insuffisant et ne peut permettre aux agents et fonctionnaires de l'Etat de satisfaire à leurs besoins vitaux (alimentation, logement, santé, éducation,...). Par contre, seuls deux enquêtés, soit 2,5\% pensent que le salaire des agents de l'Etat est décent car leur vie quotidienne en dépend largement. En relayant l'opinion de cette première catégorie d'enquêtés, il importe de dire que le barème salarial tel qu'élaboré et appliqué par l'Etat congolais ne tient pas compte des réalités socio-économiques auxquelles les 
agents et fonctionnaires de l'Etat sont confrontées. Avec ce salaire, il est difficile de consommer, d'épargner et d'investir. C'est pour cette raison que nos enquêtés (agents et fonctionnaires de l'Etat) disent qu'ils reçoivent un salaire dérisoire dénommé "SIDA» qui signifie (Salaire Insignifiant, Difficilement Acquis). Par ailleurs, comme nous l'avons vu précédemment, ces agents ne reçoivent pas de manière régulière leur salaire, car la plupart d'entre eux connaissent beaucoup d'arriérés de mois impayés par leur employeur qu'est l'Etat congolais. C'est ainsi que ce salaire met l'agent dans une double aliénation : d'une part l'aliénation vis-à-vis de son patron qui est l'Etat congolais, d'autre part l'aliénation vis-à-vis de la réalité socio-économique en face de la quelle il se trouve inadaptée. Car, non seulement ce salaire est irrégulièrement perçu mais encore il est insuffisant.

$\mathrm{Si}$ le salaire ne permet pas de répondre aux besoins fondamentaux, ce qu'il ne peut ni garantir un épanouissement de l'agent ni lui permettre d'épargner, ni moins encore d'investir pour des réalisations ultérieures. Ce qui peut l'entrainer dans un cycle de pauvreté tel que ci-dessous analysé par Ragnard Nurkse, cité par Jacques Brasseuil ${ }^{27}$ dans ce qu'il a appelé cercle vicieux de la pauvreté :

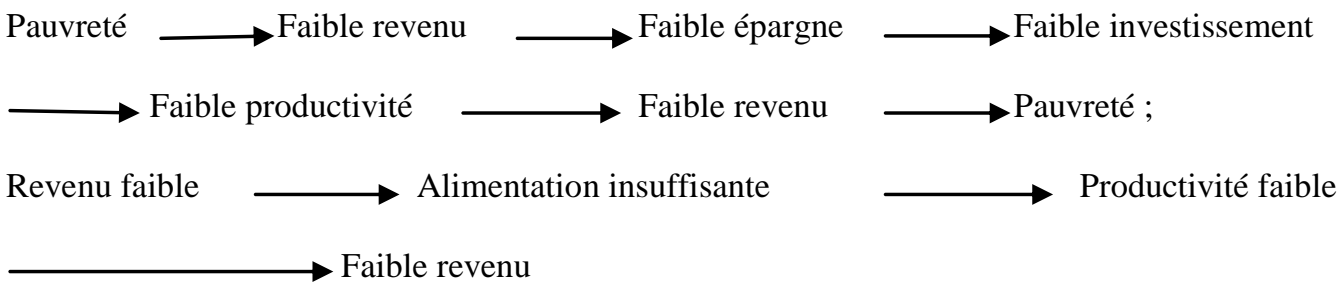

De ce schéma, il se dégage que le travail à faible revenu est source de la pauvreté, car il sera difficile pour le travailleur d'épargner, d'investir ou moins encore d'avoir une alimentation suffisante.

De cette situation, voici notre schéma triangulaire de la prolétarisation :



Nous retenons de ce triangle de prolétarisation que dans le processus de l'exploitation, le salaire insignifiant place l'agent et fonctionnaire de l'Etat dans la pauvreté ainsi que dans la misère exacerbée.

III.3. Impact du salaire des agents de l'Etat sur les conditions de vie et de travail III.3.1. La « sidatisation » du salaire par les agents et fonctionnaires de l'Etat

Comme nous l'avons démontré dans les lignes précédentes, le salaire perçu par les agents et fonctionnaires de l'Etat dans la ville de Bukavu, ne permet pas à ces derniers de consommer, d'épargner et d'investir. C'est un salaire insignifiant qu'ils reçoivent ; raison pour laquelle 97,5\% d'enquêtés le qualifient du " SIDA », c'est-à-dire Salaire Insignifiant, Difficilement Acquis. Par ailleurs, nombreux agents ne reçoivent pas régulièrement leur salaire, car ils connaissent des arriérés de plusieurs mois impayés par leur employeur qui est l'Etat congolais. Donc, avec ce salaire il est très difficile pour les bénéficiaires de s'épanouir et de bien s'intégrer dans la société.

Ainsi, après enquête, il s'avère que ce salaire a un impact néfaste sur les conditions de vie et de travail des agents et fonctionnaires de l'Etat.

${ }^{27}$ J. BRASSEUIL, Introduction à l'économie de développement, Armand Colin, Paris, 2008, p.87. 
La Politique Salariale Et La Proletarisation Des Agents Et Fonctionnaires De L'etat. (Etude ..

Tableau n ${ }^{\circ} 6$ : Impact du salaire insignifiant sur les conditions de vie de l'agent et fonctionnaire de l'Etat

\begin{tabular}{|l|c|l|}
\hline Impact du salaire dérisoire & Effectifs & Pourcentage \\
\hline La pauvreté & 50 & 62,5 \\
\hline $\begin{array}{l}\text { La corruption et détournement de } \\
\text { deniers publics }\end{array}$ & 15 & 18,7 \\
\hline $\begin{array}{l}\text { Le non-respect du temps et absence } \\
\text { au travail }\end{array}$ & 12 & 15 \\
\hline $\begin{array}{l}\text { La débrouillardise et cumule de } \\
\text { fonction }\end{array}$ & 3 & 3,7 \\
\hline Total & 80 & 100 \\
\hline
\end{tabular}

Source : nos enquêtes sur le terrain

Il ressort de ce tableau que $62,5 \%$ d'enquêtés pensent que le salaire insignifiant a comme impact la pauvreté des agents et fonctionnaires de l'Etat, la corruption et le détournement de deniers publics, soit 18,7 \% d'enquêtés ; le non-respect du temps et l'absence au travail, soit $15 \%$ d'enquêtés ; et la débrouillardise et le cumule de fonction, soit $3,7 \%$.

\section{CONCLUSION}

Une bonne politique salariale est celle qui tient compte de l'amélioration de condition de vie des travailleurs. En grande partie, les agents et fonctionnaires de l'Etat dans la ville de Bukavu sont des travailleurs pauvres. La politique salariale appliquée dans les services publics de la ville de Bukavu est une politique inadaptée aux besoins des agents et fonctionnaires de l'Etat. C'est une politique qui crée un effet de structure, c'est-à dire que les hommes politiques ont de hauts salaires par rapport à d'autres couches d'agents et fonctionnaires de l'Etat ayant de bas salaires. Ainsi, c'est une politique salariale qui prolétarise les agents et fonctionnaires de l'Etat. Elle est insignifiante et place les bénéficiaires dans une situation de paupérisation. Cela fait que nombreux agent s'adonnent à des pratiques de la corruption, le détournement de deniers publics, le retard et absence au travail,...

Par ailleurs, nombreux sont ces agents et fonctionnaires de l'Etat qui sont au service depuis plusieurs années, ils n'ont ni matricule ni payes. D'autres sont matriculés mais ne reçoivent pas le salaire, insignifiant soit-il. Compte tenu de l'ampleur du phénomène, dans le but de contribuer à l'amélioration de la situation salariale des agents et fonctionnaires de l'Etat, nous suggérons ce qui suit :

\section{1). A l'Etat}

- De mettre sur le bon pied une politique salariale adaptée aux besoins des agents et fonctionnaires

- De payer tous les agents et fonctionnaires sans discrimination

- D'encourager la justice distributive de salaires entre fonctionnaires en évitant de donner un salaire faramineux seulement à la classe politique.

\section{2). Aux agents et fonctionnaires}

- D'éviter la corruption et d'autres antivaleurs au travail

- De constituer un syndicat fort et dynamique capable de bien canaliser les besoins salariaux de membres

- Former et consolider une solidarité sociale pour bien revendiquer la paie et l'augmentation de salaire. 